\title{
Stimuli-Responsive Polymers: Design, Synthesis, Characterization, and Applications
}

\author{
Dong Wang, ${ }^{1}$ Matthew D. Green, ${ }^{2}$ Kai Chen, ${ }^{3}$ \\ Chalongrat Daengngam, ${ }^{4}$ and Yohei Kotsuchibashi ${ }^{5}$ \\ ${ }^{1}$ Department of Electrical and Computer Engineering, Virginia Tech, Blacksburg, VA 24061, USA \\ ${ }^{2}$ Department of Chemical Engineering, School for Engineering of Matter, Transport, and Energy, Arizona State University, \\ Tempe, AZ 85287-6106, USA \\ ${ }^{3}$ International Center for Material Nanoarchitectonics, National Institute for Materials Science, Tsukuba, Ibaraki 3050044, Japan \\ ${ }^{4}$ Department of Physics, Prince of Songkla University, Hatyai, Songkhla 90110, Thailand \\ ${ }^{5}$ Department of Materials and Life Science, Shizuoka Institute of Science and Technology, Fukuroi, Shizuoka 4378555, Japan
}

Correspondence should be addressed to Dong Wang; wdong@vt.edu

Received 4 July 2016; Accepted 5 July 2016

Copyright (C) 2016 Dong Wang et al. This is an open access article distributed under the Creative Commons Attribution License, which permits unrestricted use, distribution, and reproduction in any medium, provided the original work is properly cited.

\begin{abstract}
Stimuli-responsive polymers or so-called "smart polymers" are macromolecules that are sensitive to certain triggers from the external environment, including temperature, light, electrical or magnetic fields, and chemicals. The activated polymers produce observable or detectable micro- or nanoscale changes, such as morphology, molecular bond rearrangement/cleavage, and molecular motion, which can induce changes in their macroscopic properties such as color, shape, and functionality. Due to the versatile selection of backbone and functional groups, stimuli-responsive polymers can be tailored to have a variety of specific mechanical, chemical, electrical, optical, biological, or other properties and can be engineered into different forms, including bulk, thin film, micro/nanoparticles, and composites. Over the years, many multidisciplinary efforts have been conducted and reported optimizing the functionality of stimuli-responsive polymers and exploring new and innovative applications. However, as shown below, original and exciting research in emerging sectors continues to drive the evolution of and interest in this class of polymer.

After a rigorous peer review process, eight articles were compiled into this special issue as an effort to represent the most recent developments in this field. A variety of topics are included in this special issue such as experimental and theoretical modeling efforts with a range of applications, including sensing, biomedical devices, polymer layers,
\end{abstract}

hydrogels, nanocomposites, shape-memory polymers, and polymer gels for agriculture.

Tuning the adhesion properties of polymers, surfaces, and hydrogels plays a very important role in fields such as membrane technology, biomanufacturing, and tissue engineering. In the paper titled "Combinational Effect of Cell Adhesion Biomolecules and Their Immobilized Polymer Property to Enhance Cell-Selective Adhesion" polymeric substrates with preselected hydrophobicity are modified with biomolecules to promote cell-selective adhesion. These materials offer great potential for tissue engineering and cell sorting technologies. Similarly, the adsorption characteristics of a polymer gel are studied in the paper titled "Protein Adsorption on Hybrids of Thermoresponsive Polymers and Single-Walled Carbon Nanotubes." The authors investigate the random adsorption of RecA proteins onto hybrids containing PNIPAm and singlewalled carbon nanotubes (SWNTs) while in the presence or absence of DNA molecules. The thermoresponsive character of PNIPAm is used to regulate protein adsorption. These findings open the door to the nanobiodevices using PNIPAmSWNT hybrids.

Developments in the field of hydrogels for wound healing and drug delivery have revolutionized approaches to treating chronic skin wounds, cancer therapies, and many other diseases. The paper titled "Fabrication of HyaluronanPoly(vinylphosphonic acid)-Chitosan Hydrogel for Wound 
Healing Application" reports the fabrication and characterization of a new hydrogel with a three-dimensional highly porous structure, short gelation time, high degradation rate, and the ability to prevent E. coli infections. Such a hydrogel has great potential to be used as bioadhesive for skin wound healing applications. Also, the paper titled "Incorporation of Amphipathic Diblock Copolymer in Lipid Bilayer for Improving $\mathrm{pH}$ Responsiveness" studies how changes in environmental $\mathrm{pH}$ cause a change in the bilayer fluidity, which regulates the release rate of doxorubicin. Such improved $\mathrm{pH}$-controllability over the drug release from hybrid liposomes offers new insights into the design of drug delivery systems. Another drug delivery approach utilizing stimuliresponsive polymers is reported in "Magnetically Triggered Monodispersed Nanocomposite Fabricated by Microfluidic Approach for Drug Delivery." In this paper, a nanocomposite gel consisting of PNIPAm, which is a gel that can swell or shrink in response to temperature or $\mathrm{pH}$, and magnetic iron oxide nanobeads is developed for controlled release of liquid drugs upon exposure to an alternating magnetic field. Drug release from such nanocomposites can be remotely triggered by a magnetic field and heated through magnetic losses, which presents an innovative strategy for targeted and usercontrolled drug delivery.

The properties of stimuli-responsive polymers can also be harnessed for sensing applications. For example, the paper titled "Electrospun PEDOT:PSS/PVP Nanofibers for CO Gas Sensing with Quartz Crystal Microbalance Technique" reports on the effects of the surface area of PEDOT:PSS/PVP nanofiber membranes on the adsorption of CO. The adsorption process triggers a change in the oscillation frequency, which enables sensitivity to low concentration (5-50 ppm) of $\mathrm{CO}$ with a linear relation to the resonant frequency.

Modeling and simulating polymeric systems provide critical information about the molecular processes that govern polymer properties and phenomena. These powerful tools can be used to study a great variety of polymer properties, systems, and behaviors. In the paper "Modeling Based Characterization of Thermorheological Properties of Polyurethane ESTANE ${ }^{\mathrm{TM}}$ " the commercially available shapememory polymer, ESTANE, is investigated using Dynamic Mechanical Thermal Analysis (DMTA). The coefficient of thermal expansion above and below the glass transition temperature is estimated and a finite viscoelastic and incompressible material model is used to predict the viscoelastic properties observed in the uniaxial stress relaxation experiments. This work may provide the necessary knowledge base for further progress in the development of shape-memory polymers. Also, modeling stimuli-responsive polymers can shed light on agricultural and biological research. An example is demonstrated in the paper titled "Phase Characterization of Cucumber Growth: A Chemical Gel Model." The effect of hydrogel swelling in both pre- and postharvest stages on cucumber growth is studied. In fruit development, the hormones stimulate the expansion of cells, which is similar to the swelling mechanism of the hydrogel. The model offers solutions to regulate fruit development and can be extended to other plant candidates for potential biological and food engineering.
We believe the research reported in this issue can enrich the basic understandings and stimulate further development of these advanced smart materials.

\section{Acknowledgments}

We appreciate the great efforts and contributions from the authors and reviewers to the special issue.

Dong Wang

Matthew D. Green

Kai Chen

Chalongrat Daengngam

Yohei Kotsuchibashi 

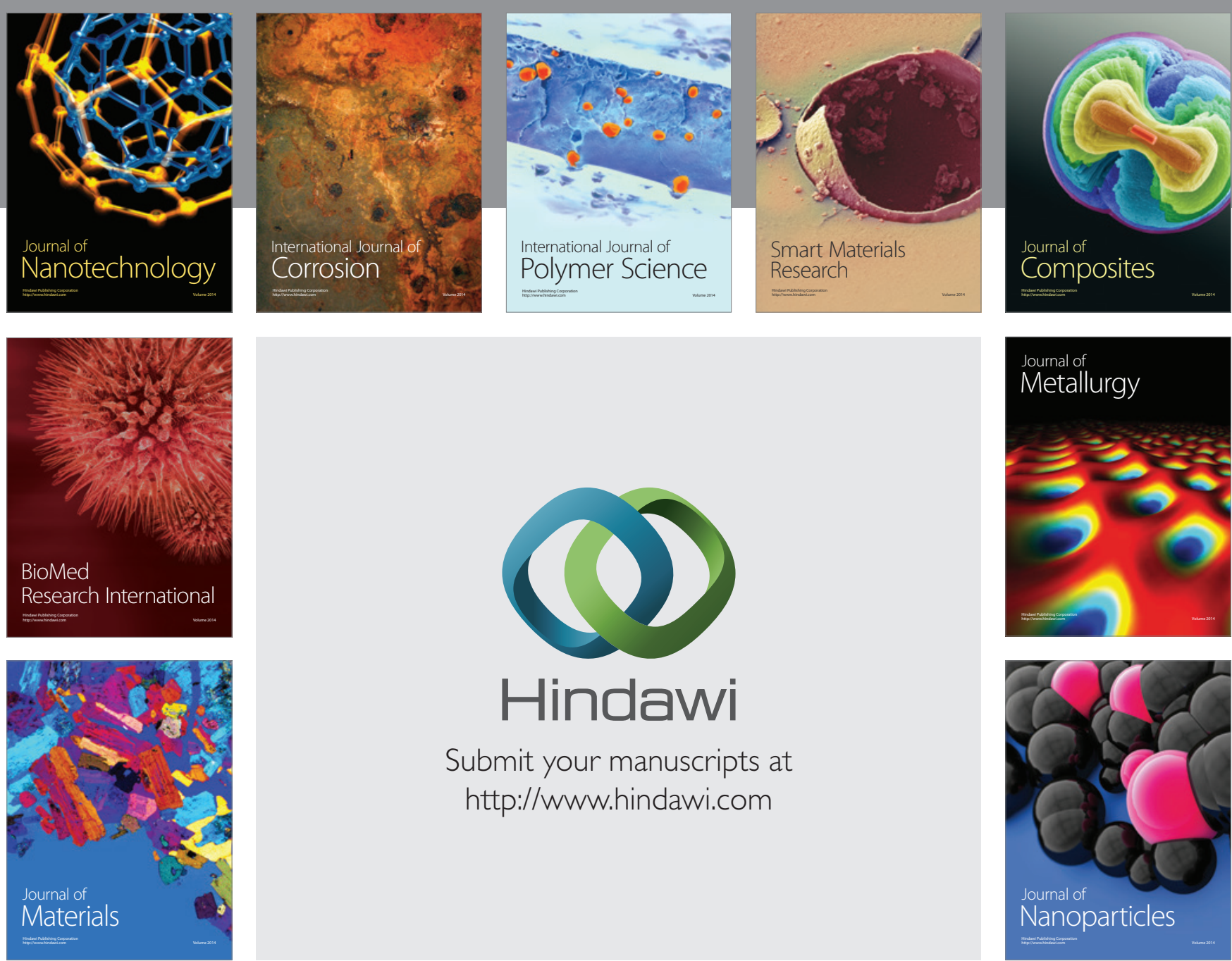

\section{Hindawi}

Submit your manuscripts at

http://www.hindawi.com

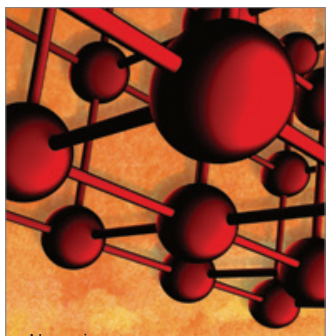

Materials Science and Engineering
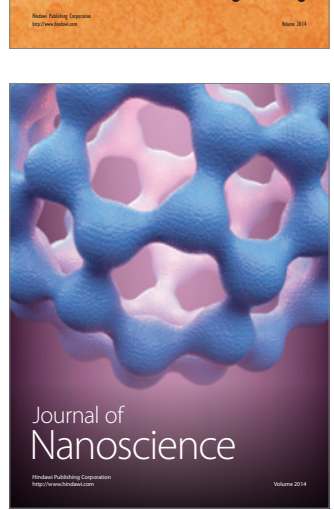
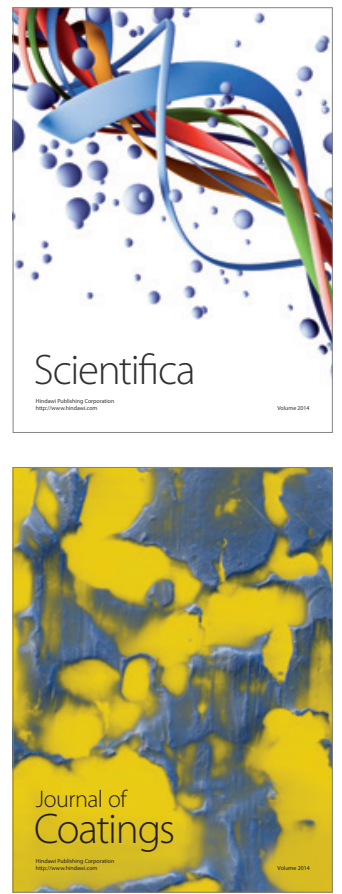
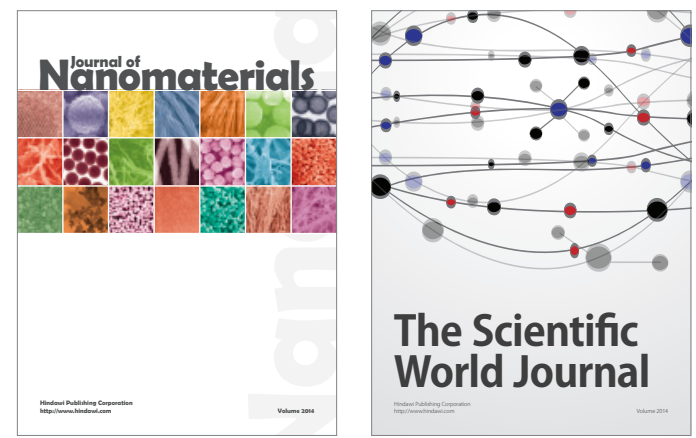

The Scientific World Journal
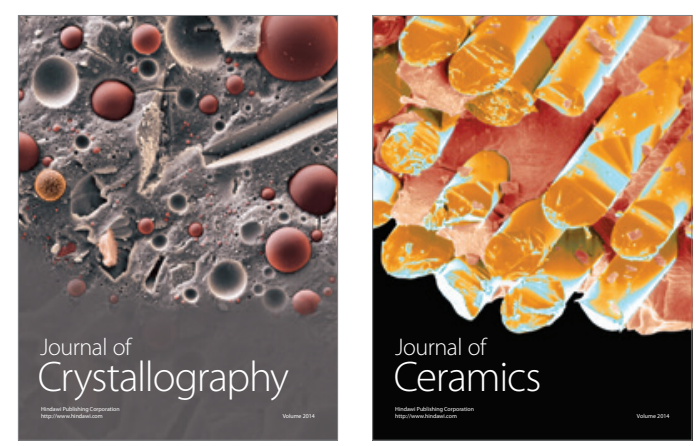
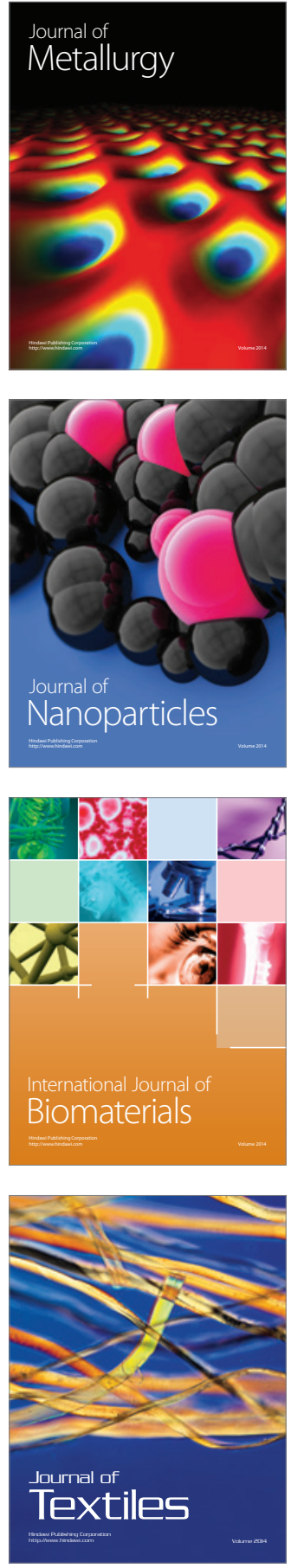